\title{
Sex preference and body orientation preference in huddling behavior of rat pups
}

\author{
RICHARD DENI, ROBYN MARTIN, JENNIFER WHEELER MAKIN, \\ JOSEPHINE LONGO, and SUZANNE ANDRES \\ Rider College, Lawrenceville, New Jersey 08648
}

\begin{abstract}
The social huddling of six litters of rat pups (Rattus norvegicus) was observed on Days 6-9 postpartum without the dam present. Each litter was reduced to three male and three female pups. Thirty-six pups were used as subjects (18 male, 18 female). Tests for the effects of sex of subject, age of subject, sex of littermate huddled against, and body orientation during huddling were carried out. Results indicated that subjects huddled against opposite-sex littermates more frequently than against same-sex littermates. Huddling was a curvilinear function of age, with average frequency of huddling reaching a peak on the second day of observation (Day 7 postpartum) and decreasing thereafter. Results also showed that subjects, regardless of sex, huddled using an orientation that allowed their heads to touch more frequently than an orientation in which the head was touching the body of a sibling. These data on early sex preference and body orientation preferences during huddling suggest that rat pups are competent in aspects of behavior having attachment potential. The findings also help define the age of social competence in this species.
\end{abstract}

Recent research on the development of social attachments in altricial species has focused on aspects of attachment potential derived from the separate contributions of parent and offspring. Altricial development implies that early attachment potential related to parent-offspring bonds as well as to sibling bonds results from parental competence. Only as the offspring grow older do they contribute to the development of attachments. For example, previous work has set certain limits on the ability of rat pups to make social preference responses. Gregory and Pfaff (1971) reported that reliable bedding-odor discriminations and preferences did not occur prior to 12 days of age. Also, diet-dependent conspecific odor preferences have been documented in rat pups no younger than 20 days postpartum (Leon, Galef, \& Behse, 1977). Additional "age thresholds" have been reported for the social huddling behavior of single isolated rat pups (Alberts, 1978b): Only pups older than 15 days spend more than $50 \%$ of a test session huddling with an anesthetized target pup in the center of an enclosure; only pups older than 10 days spend more than $50 \%$ of a test session huddling with a dead ambient-temperature pup; pups as young as 5 days postpartum spend $50 \%$ or more of a test session huddling with a warm loop of tubing; only pups 10 days or older show disruption of huddling with a single anesthetized pup after intranasal zinc sulfate treatment.

Address reprint requests to Richard Deni, Psychology Department, Rider College, Box 6400, Lawrenceville, New Jersey 08648. We thank Eileen Manela for her assistance.
Evidence is accumulating to support the view that offspring competencies having attachment potential are present during the early period of 5-10 days postpartum (cf. Kleitman \& Satinoff, Note 1). Alberts (1978a) has reported that 5-day-old litters use thermal cues to regulate their position within a clump when tested in isolation from a dam. Alberts (1978b) has also shown that 5-day-old pups orient toward a warm stimulus object (loop of tubing), further confirming their ability to use thermal cues in the execution of huddling responses in the absence of the dam. Further questions remain concerning the range of cues, both social and nonsocial, that can be used by 5-10-dayold pups to control their social huddling responses. The present study addresses the question of whether pups in this age range are aware of static stimulus factors such as littermate gender and littermate body orientation. Such an awareness would be indicated if rat pups demonstrate a preference for huddling with same- vs. opposite-sex littermates and demonstrate a tendency to huddle in a specific body orientation.

\section{METHOD}

\begin{abstract}
Subjects
Six litters of Norway rats (Rattus norvegicus) were used as subjects. To obtain these litters, six nulliparous adult female rats were selected at random and were mated with six randomly selected adult males with previous mating experience. The dams were housed with their litters in a large colony room (lights on at 0700 , off at $1900 \mathrm{~h}$ ) in standard rectangular plastic cages with wire covers. Pine shavings were used as bedding material. Rat chow and water were freely available in the home cage. On Day 1 postpartum, each litter was reduced to three male subjects and three female
\end{abstract}


subjects by random selection. These manipulations resulted in two groups of subjects: 18 male pups and 18 female pups. No further handling occurred until Day 5 , when each pup was removed from the home cage and a drawing of the dark pigment pattern on the dorsal body surface was made to code individual pups for identification.

\begin{abstract}
Apparatus
During each observation, a given litter was transferred from the home cage to another standard rectangular plastic cage without a cover. The observation cage was washed and filled with clean shavings prior to each session. A black-and-white video camera was positioned above the observation cage to record the huddling contacts of the six subjects within each litter. Observations were taped on a videocassette recorder located in another room. All videotaping took place in a small room apart from the colony room, in which temperature was maintained at $72^{\circ} .73^{\circ} \mathrm{F}$, and standard overhead lighting was provided by two $40-\mathrm{W}$ fluorescent tubes.
\end{abstract}

\section{Procedure}

Four daily videotapings were made for each litter (three male, three female subjects) starting on Day 6 postpartum. Litters were videotaped for 150 min beginning at $1100 \mathrm{~h}$. Just prior to each session, the pups were separated from the dam and were labeled by painting the top of their heads with white typing correction fluid using a dot pattern code (one to six dots, $3 \mathrm{~mm}$ in diameter). The litter was then placed in the observation cage and was positioned under the video camera. The video recording method involved taping for $1 \mathrm{~min}$ every $9 \mathrm{~min}$ to compress the 150 -min session into 15 consecutive 1-min episodes. These 1-min episodes were used as samples of behavior and were coded by the experimenters. The following huddling data were recorded during tape playback: number of touching contacts made by each of the six pups (in each of six litters) with any littermate of the same and opposite sex, and number of touching contacts made by each pup with littermates in a head-to-head touching orientation and headto-body orientation. Each videotape record provided 15 views of the clump containing the six subjects (three male, three female). Repeated passes through the episodes were made, with each litter member as the target subject. Recording of randomly selected individual records was done periodically to assess interobserver reliability.

\section{RESULTS}

Assessment of reliability resulted in percent agreement scores above $81 \%$ on all measures. Sex preference data and body orientation preference data were each analyzed with a three-factor mixed analysis of variance. In each case, sex of subject was the between-subjects factor, and age of subject was a within-subjects factor. The second repeated-measures factor was either category of littermate huddled against (same- vs. opposite-sex) or category of body orientation during huddling (head-to-head vs. head-to-body).

\section{Sex Preference}

The top panel of Table 1 shows the average number of same-sex and opposite-sex contacts per session (a session consisted of 151 -min episodes). Significantly more opposite-sex contacts were recorded $[F(1,28)=$ 84.83, $\mathrm{p}<.001$ ]. The bottom panel of Table 1 shows the average number of huddling contacts as a function of age of subject. The main effect for age of subjects was significant $[F(3,84)=7.02, p<.001]$.
Table 1

Means and Standard Deviations for Huddling Contacts per Session as a Function of Sex of Littermate and Age of Subject

\begin{tabular}{ccc}
\hline Condition & Mean & SD \\
\hline & Main Effect: Sex of Littermate \\
Same Sex & 7.4 & 3.8 \\
Opposite Sex & 12.1 & 4.9 \\
& Main Effect: Age of Subject \\
Day 6 & 8.2 & 4.7 \\
Day 7 & 11.4 & 4.7 \\
Day 8 & 10.1 & 3.8 \\
Day 9 & 9.2 & 4.6 \\
\hline
\end{tabular}

Table 2

Means and Standard Deviations for Huddling Contacts per Session for the Interaction of Sex of Littermate and Age of Subject

\begin{tabular}{ccrr}
\hline Day & Sex of Littermate & Mean & SD \\
\hline \multirow{2}{*}{6} & Same & 6.1 & 4.3 \\
& Opposite & 10.2 & 5.8 \\
7 & Same & 7.9 & 2.8 \\
& Opposite & 14.9 & 3.6 \\
8 & Same & 8.1 & 3.4 \\
\multirow{2}{*}{9} & Opposite & 12.1 & 3.2 \\
& Same & 7.3 & 4.3 \\
& Opposite & 11.1 & 4.0 \\
\hline
\end{tabular}

Post hoc Newman-Keul's tests showed that a significant increase $(p<.05)$ in huddling contacts occurred from Day 6 to Day 7, and a significant decrease $(p<.05)$ occurred from Day 7 to Day 9. Table 2 shows the average number of huddling contacts as a function of the interaction of sex of littermate huddled against and age of subject. The interaction effect was significant $[\mathrm{F}(3,84)=3.24, \mathrm{p}<.025]$. Post hoc Newman-Keul's tests showed that oppositesex contacts were significantly more frequent $(\mathrm{p}<.05)$ than same-sex contacts at each age level.

\section{Body Orientation Preference}

The average number of huddling contacts in the head-to-head orientation (male and female data combined) was 11.6 (standard deviation $=6.1$ ) and for the head-to-body orientation was $\mathbf{2 . 3}$ (standard deviation =3.9). There were significantly more head-to-head contacts $[F(1,28)=70.43, p<.001]$.

\section{DISCUSSION}

Characteristic interpretations of altricial development place heavy emphasis on parental care as a precursor for the development of social behavior. According to this view, contacts between offspring and mother are physically maintained by the mother (through nesting, retrieval, and pup licking), and numerous studies of maternal behavior in rats have 
been reported. On the other hand, relatively little is known of the contribution of the offspring themselves to the formation of early sibling attachments in altricial rodents or even if early (5-10 days of age) attachments exist. Alberts and Brunjes (1978) have advanced an explanation of the development of huddling in rat pups based on a two-phase process. Early development of huddling in 5-10-day-old pups, according to this view, is controlled by physiological factors including, primarily, responsiveness to thermal cues. Five-day-old pups preferred to huddle against a warm inanimate stimulus rather than a conspecific, whereas older pups preferred the conspecific. Alberts and Brunjes described this transition as an indication of the development of "filial" huddling controlled by responsiveness of species odor.

The data reported here show that 6-9-day-old rat pups demonstrate a preference for huddling against opposite-sex littermates and huddling in a head-to-head (heads touching) body orientation. These findings indicate that "filial" huddling might also be mediated by sex-specific cues, although a sex-odor explanation seems unlikely, based on the body orientation preference observed in the present study. The body orientation preference could be explained in terms of the development of tactile sensitivity in a cephalocaudal progression. Rat pups might huddle head-to-head because the head region is maximally sensitive to tactile inputs. On the other hand, the maintenance of an opposite-sex huddling preference could be explained based on the transmission and uptake of sex-specific cues. Odor cues related to elimination are not likely candidates, since pups, on the average, did not orient their olfactory receptors to the ano-genital region of littermates during huddling (pups preferred a headto-head posture). In addition, active elimination of wastes by pups would require oral stimulation of the ano-genital region in a manner characteristic of mother-pup interaction but not of pup-pup interaction at 6-9 days postpartum.

It is possible that the pups in this study were able to discriminate one another based on behavioral cues. Such cues might include activity patterns, posturing, and ultrasound vocalizations. These cues, if they are present, would not have been available to subjects in the studies reported by Alberts and Brunjes, since pups in those studies were tested individually in choice tests involving immobile target pups (cf. Brunjes \& Alberts, 1979).

\section{REFERENCE NOTE}

1. Kleitman, N., \& Satinoff, E. Thermoregulatory behavior in newborn rats. Paper presented at the annual meeting of the International Society for Developmental Psychobiology, 1980.

\section{REFERENCES}

AlBerts, J. Huddling by rat pups: Group behavioral mechanisms of temperature regulation and energy conservation. Journal of Comparative and Physiological Psychology, 1978, 92, 231245. (a)

AlBERTs, J. Huddling by rat pups: Multisensory control of contact behavior. Journal of Comparative and Physiological Psychology, 1978, 92, 220-230. (b)

Alberts, J., \& Brunjes, P. Ontogeny of thermal and olfactory determinants of huddling in the rat. Journal of Comparative and Physiological Psychology, 1978, 92, 897-906.

Brunjes, P., \& Alberts, J. Olfactory stimulation induces filial preferences for huddling in rat pups. Journal of Comparative and Physiological Psychology, 1979, 93, 548-555.

Gregory, E., \& Pfaff, D. Development of olfactory-guided behavior in infant rats. Physiology \& Behavior, 1971, 6, 573-576.

Leon, M., Galef, B., \& Behse, J. Establishment of pheromonal bonds and diet choice in young rats by odor pre-exposure. Physiology \& Behavior, 1977, 18, 387-391.

(Received for publication November 14, 1980; revision accepted February 10, 1981.) 